\title{
Transport in tight-binding bond percolation models
}

\author{
Daniel Schmidtke, ,, A Abdellah Khodja,, , 7 and Jochen Gemmer 1 , 团 \\ ${ }^{1}$ Fachbereich Physik, Universität Osnabrück, Barbarastrasse 7, D-49069 Osnabrück, Germany
}

\begin{abstract}
Most of the investigations to date on tight-binding, quantum percolation models focused on the quantum percolation threshold, i.e., the analogue to the Anderson transition. It appears to occur if roughly $30 \%$ of the hopping terms are actually present. Thus, models in the delocalized regime may still be substantially disordered, hence analyzing their transport properties is a nontrivial task which we pursue in the paper at hand. Using a method based on quantum typicality to numerically perform linear response theory we find that conductivity and mean free paths are in good accord with results from very simple heuristic considerations. Furthermore we find that depending on the percentage of actually present hopping terms, the transport properties may or may not be described by a Drude model. An investigation of the Einstein relation is also presented.

PACS numbers: $\quad 05.60 . \mathrm{Gg}, 72.80 . \mathrm{Ng}, 66.30 . \mathrm{Ma}$,
\end{abstract}

\section{INTRODUCTION}

Percolation theory is a well known method to describe transport properties of crystals or other systems which feature regular lattices with substantial amounts of defects, impurities etc. It has been vastly studied from a classical point of view [1, 2]. Here usually bonds (or sites) are filled at random on the above lattice with a probability $p$. It turns out that, depending on the type of lattice, there exists some $p$ at which the probability of getting a connected cluster of bonds (sites) which extends through the whole lattice changes abruptly from approximately zero to approximately one. This $p$ is called the critical probability $p_{c}$.

Due to increasing interest in microscopic structures, which may be significantly affected by quantum effects, percolation models based on quantum mechanics, have also received considerable attention. Also genuinely quantum phenomena like, e.g., quantum hall effect [3], Fermi-Bose mixtures [4] or general (anti)ferromagnetic systems [5], have been addressed by means of percolation theory.

The main focus of the literature on quantum percolation appears to be on the transition from the "non-transport" to the transport regime which is essentially of the same type as the the well-known Anderson transition [6, 7]. Much effort is dedicated to the determination of the quantum percolation threshold $p_{q}$. It is found that the quantum percolation threshold is greater than the classical one [6, 8, 9]. For example the classical threshold for bond percolation in three dimensions on a simple cubic lattice is determined to be $p_{c}^{b} \approx 0.25$ [2, 10] whereas the threshold for quantum percolation has been determined to lie at $p_{q} \approx 0.31[8,11]$. However, quantitative investigations of transport in the delocalized regime appear to be restricted to preliminary studies close to the quan-

\footnotetext{
*Electronic address: danischm@uos.de

${ }^{\dagger}$ Electronic address: akhodja@uos.de

‡Electronic address: jgemmer@uos.de
}

tum percolation threshold [6, 8, 11]. With the work at hand we aim in contrast at a more detailed understanding of transport in systems whose structures are on one hand far away from clean crystals but on the other also far from being collections of disconnected clusters. More specifically, we quantitatively address transport properties at bond probabilites $p$ at which almost all energy eigenstates are delocalized. Generally we expect (and find) diffusive behavior. Unlike the diffusive behavior in periodic systems which is restricted to finite time (and length) scales [12 14 the diffusive behavior here persists due to broken translational symmetry.

The primary motivation for the work at hand is of principal and theoretical nature. Just like it has been recently done for the Anderson model [15], we intend to demonstrate that also in percolation models regular diffusive behavior must not necessarily be induced by decoherence sources like phonon-coupling, etc., but may emerge in a fully coherent set up from the electronic model itself. Furthermore, even in the absence of decoherence this transport behavior will be demonstrated to be in good accord with simple statistical descriptions like the Drude-model. However the considerations are not entirely detached from concrete experimental research. In the context of research based on ultra-cold atoms the dynamics of a moderate number of atoms (playing the role of electrons) subject to a trap and an underlying optical lattice (but completely isolated from any environment otherwise) is observed. Among the central questions are the transport properties of such coherent systems. [16, 17] The percolation models we address below may also possibly be implemented within such an an experimental framework through a modification of the optical lattice. In this context bond percolation may be more convenient to implement then site percolation. Furthermore, in the context of real materials, percolation models may be very rough descriptions of binary mixed crystal alloys in which the on-site potential of one species exceeds the band with of a regular crystal formed by the other species. In this case the lattice would separate in two sub-lattices, each formed by sites occupied by the the same species only. This would correspond to site perco- 
lation rather than bond percolation, however transport properties may be expected to behave similarly. Recent investigations on magnesium alloys indicate an massive increase of resistivity caused by the substitution of only a few percent of the sites by another species, this being in accord with the findings in the paper at hand [18]. This is discussed in more detail in Sec. VI

The paper at hand is organized as follows. In Sec. II we introduce our one-particle, tight-binding percolation model and comment rather briefly on localization using the density of states and the inverse participation number in Sec. III Thereafter (Sec. IV) we specify the quantities of interest, namely the dc-conductivity and current auto-correlation function as connected by linear response theory. We address those quantities numerically and employ a method based on "quantum typicality" whenever samples are required that are too large to be assessed by means of exact diagonalization. We find hints of a transition from "non- Boltzmann" to Boltzmann-type transport with increasing $p$. Section $\nabla$ establishes the validity of the Einstein relation and, based on the latter introduces a mean free path. A numerical investigation of this mean free path confirms the above "non- Boltzmann" to Boltzmann-transport transition. Section VI is dedicated to a comparison of our results to experimental data on binary magnesium alloys. The paper closes with summary and conclusions in Sec. VII]

\section{TIGHT-BINDING BOND PERCOLATION MODEL}

The field of percolation models includes a vast number of various approaches to describe processes in semiconductors or other disordered materials. A general division is given by the description of defects, or whatever is causing the disorder, either by loss of particles (site percolation) or loss of bonds between sites (bond percolation), whereby instead of loss one can observe various bond-strength or energies at the sites as well [6, 8].

In the paper at hand we investigate transport in bond percolation models. The intention here is not the detailed description of any specific material but rather the overall description of transport in quantum models of the percolation type. Therefore we consider in the following a three dimensional cubic lattice with edge length $L$, i.e., the total number of sites (or quantum-dimension of the Hamiltonian) is $\operatorname{dim}\{\hat{H}\}=\left(\frac{L}{a}\right)^{3}=N$, where $a$ denotes the lattice constant, i.e., the distance between neighbouring sites.

Generally (quantum) one-particle, tight-binding bond percolation may be described by

$$
\hat{H}=\sum_{<i j>} t_{i j} \hat{a}_{i}^{\dagger} \hat{a}_{j}
$$

where $\langle i j\rangle$ denotes the summation over next neighbors and $t_{i j}$ is known as transfer amplitude. This amplitude

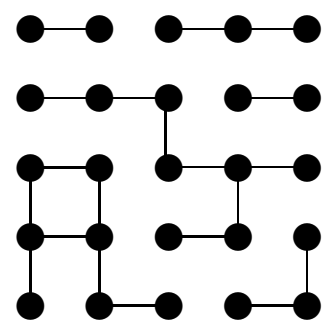

Figure 1: Two dimensional model of classical bond percolation with $p=0.5$. In classical considerations this describes the situation right at the percolation threshold, whereas from a quantum point of view this model would be below the quantum percolation threshold, even in a three dimensional version. This is due to an effect comparable to Anderson localization.

may given by

$$
t_{i j}=\left\{\begin{array}{cl}
t \exp \left(-2 \pi i \phi_{i j}\right) & \text { for connected bond } \\
0 & \text { for disconnected bond }
\end{array}\right.
$$

Here, $t$ denotes a parameter which quantifies the hoping strength and $\phi_{i j}$ denotes a parameter which may describe interactions with an external field, e.g., magnetic fields; then $\phi_{i j}$ is the Peierls phase [6]. However, for simplicity and in order to guarantee time reversibility we set $\phi_{i j}=0$. Moreover the on-site potential is set to zero for all sites, i.e. $t_{i i}=0$. Note that we also set in all calculations $k_{B}=1$ and $\hbar=1$.

The distribution of the bond "strenghts" (one for connected, zero for disconnected) is given by

$$
P\left(t_{i j}\right)=p \delta\left(t_{i j}=t\right)+(1-p) \delta\left(t_{i j}=0\right) .
$$

Fig. (1) shows an two dimensional sketch of bond percolation $p=0.5$. In the classical case one would certainly say that the percolation threshold is just reached, but as found in in [8, 9, 19] the quantum threshold is (possibly against a naive guess) higher than the classical threshold, i.e., quantum transport would be not possible in the above model, even if it was three-dimensional.

\section{PRELIMINARY INVESTIGATION OF LOCALIZATION}

Below (Sec. IV) we compute conductivities in the high temperature limit, i.e., all energy regimes contribute to transport. In such a setting an decrease of the conductivity with decreasing $p$ may indicate both, either an increase of resistivity in the delocalized energy regime, or simply an increase of the fraction of localized energy eigenstates. Since we are primarily interested in the former we perform in the following a rough analysis of the fraction of delocalized states for different $p$ 's. Then we concentrate on the regime in which the vast majority of the energy eigenstates is delocalized. Generally the precise calculation of mobility edges is a challenge that is 
dealt with using sophisticated methods. 20 22]. For the purposes at hand, however a rather rough determination of the mobility edge suffices. To this end we follow the general approach presented in [23].

The investigation at hand is based on the inverse participation number (IPN)

$$
I\left(E_{n}\right)=\sum_{i}\left|\psi_{i}\left(E_{n}\right)\right|^{4} \quad,
$$

where $\psi_{i}\left(E_{n}\right)$ denotes the the $i$-th component of the energy eigenstate corresponding to $E_{n}$. As described in 23] a convenient way to find the mobility edge is to plot the IPN at a given energy against the system size $L$ on a doubly-logarithmic scale. In this representation graphs corresponding to localized states are expected to "bend upwards" while graphs corresponding to extended states are expected to "bend downwards". Only the logarithmic IPN's at the mobility edge are supposed to form a straight line, i.e., the inverse participation number is expected to scale as

$$
I\left(E_{c}\right) \propto L^{-d_{2}},
$$

where $E_{c}$ denotes the critical energy (mobility edge) and $d_{2}$ is referred to as the fractal dimension. Though the exact determination of fractal dimensions is beyond the scope of this paper, we note that at $p=0.38$ the fractal dimension in our model is approximately given by $d_{2} \approx$ 1.6 (cf. Fig (2)). This value stands in accord with results of a recent work [24] where the critical exponent for the localization length is $d=1.627 \pm 0.055$.

Fig. (2) shows some of the described scaling graphs for various energies for $p=0.38$, all calculated by means of direct numerical diagonalization. This Fig. suggests that the mobility edge is around $E \approx 1$ (since $E=1$ appears to correspond to the straightest line). In accord with an overall symmetry of the spectrum w.r.t. energy (see Fig. (3)) we find the second mobility edge at $E=-1$. For later purpose it is useful to calculate the density of states (DOS) since it will allow us to estimate the energy range in which most energy eigenstates are delocalized. To that end we define the portion of delocalized eigenstates w.r.t. all eigenstates between the above calculated mobility edges which we denote by $\Phi(p)$.

$$
\Phi(p)=\int_{\Delta E} \rho(E) d E
$$

Before we proceed and introduce the main purpose of this work, we would like to have a closer look at the density of states for consistency of the given results.

One finds that for few impurities, i.e. $p>0.65$, the density of states is smooth and the graph is well described by a Gaussian function, regardless some peaks which correspond to special cluster configurations within the system, cf. [9, 24].

At one hand one finds for low $p$ 's that the peaks become more visible, at the other hand one notices a dip around the energy $E \approx 0$, which becomes more significant for decreasing $p$ 's. For visualization of that fact we calculated the density of states for $p=0.45$. The results are presented in Fig. (3).

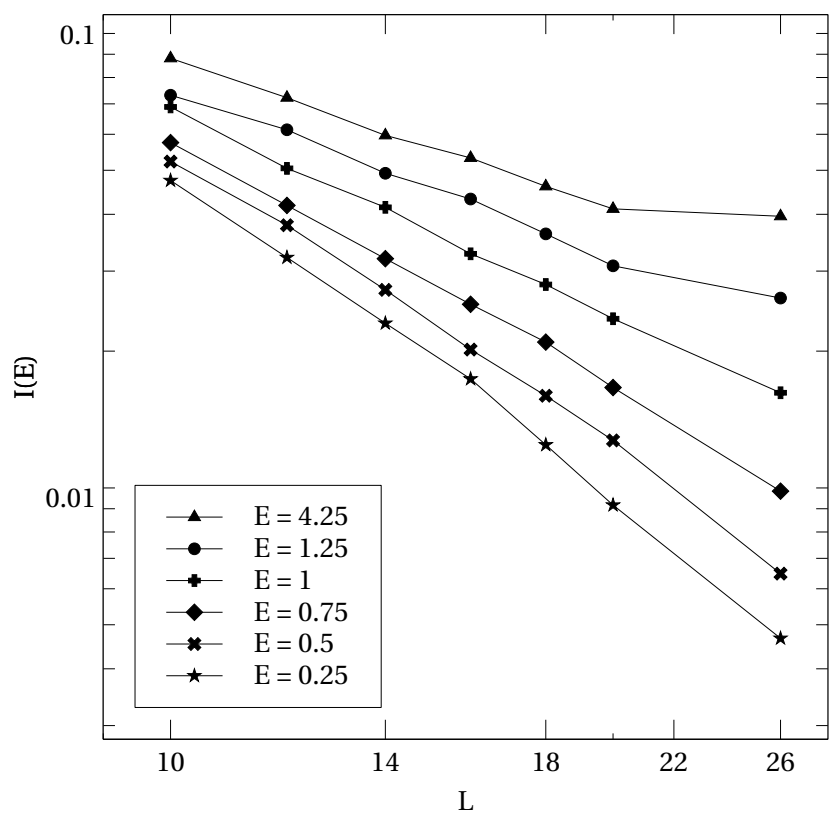

Figure 2: Scaling of the IPN with system size $L$ at several energies and percolation ratio $\mathrm{p}=0.38$. Since only at the mobility edge the scaling is allover linear we locate the mobility edges roughly at $E=-1$ and $E=1$. Only the last one $E=1$ is actually displayed above.

The results are displayed in Fig. 4, From the latter it is obvious that the regime in which the vast majority of eigenstates are delocalized is bound from below by $p \approx 0.5$. Furthermore the data appear to be in accord with the value of $p_{c} \approx 0.31$ from the literature for the Anderson transition.

\section{CURRENT DYNAMICS AND CONDUCTIVITY}

As already stated our primary interest in the paper at hand is (other than in many works in the respective literature) not the determination of the quantum percolation threshold, rather it is quantitative description of transport behavior well above that threshold, i.e., in a regime where the vast majority of states are extended. We aim at finding the dc-conductivity $\sigma_{d c}$ from linear response theory (Kubo formula) which amounts to the calculation of the particle-current autocorrelation function.

We restrict ourselves here to the limit of high temperatures and low fillings. Therefore the framework of the 


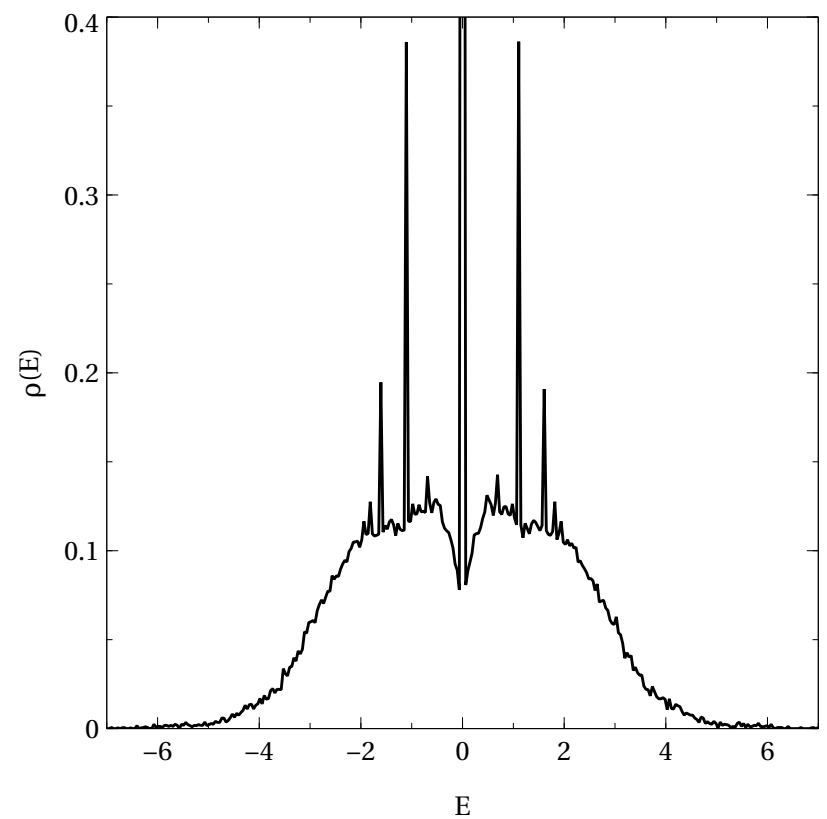

Figure 3: The density of states, here for $p=0.45$, is symmetrical w.r.t. the energy $E=0$. There are several distinct peaks, namely at $E=0$, which correspond to special cluster configurations, cf. [9]. Also notable is the dip around $E=0$, that only occurs at low $p$.

grand canonical ensemble is used [25 28] which results in

$$
\sigma_{d c}=\sigma(t \rightarrow \infty), \quad \sigma(t)=\frac{f}{k_{B} T} \int_{0}^{t} \frac{1}{V} \operatorname{Tr}\left\{\hat{J}\left(t^{\prime}\right) \hat{J}(0)\right\} d t^{\prime}
$$

Here $f$ denotes the filling factor, i.e. the number of particles per site at equilibrium, and $\hat{J}(t)$ denotes the current operator in the Heisenberg picture. Furthermore $T$ is the temperature and $k_{B}$ is the Boltzmann constant. The volume of the system is denoted by $V=a^{3} N$.

In order to employ (7) we need to specify an adequate current operator. In the context of periodic systems this is often done using a continuity equation for the siteprobabilities 29 31. Since we do not have fully evolved periodicity here we follow [32] in starting from a velocity operator instead. The velocity operator ( corresponding to motion in $\mathrm{x}$-direction) reads:

$$
\hat{v}=\frac{i}{\hbar}[\hat{H}, \hat{x}] \quad,
$$

where $\hat{x}$ denotes the $\mathrm{x}$-position operator $\left(x_{i}\right.$ denotes the $\mathrm{x}$-coordinate of the $\mathrm{i}$-th site)

$$
\hat{x}=\sum_{i=1}^{N} x_{i} \hat{n}_{i} \quad \hat{n}_{i}:=\hat{a}_{i}^{\dagger} \hat{a}_{i} \quad x_{i}=i a .
$$

Thus, the velocity operator $\hat{v}$ reads

$$
\hat{v}=\frac{i}{\hbar} \sum_{i j}^{N}(i-j) a t_{j i} \hat{a}_{j}^{\dagger} \hat{a}_{i}
$$

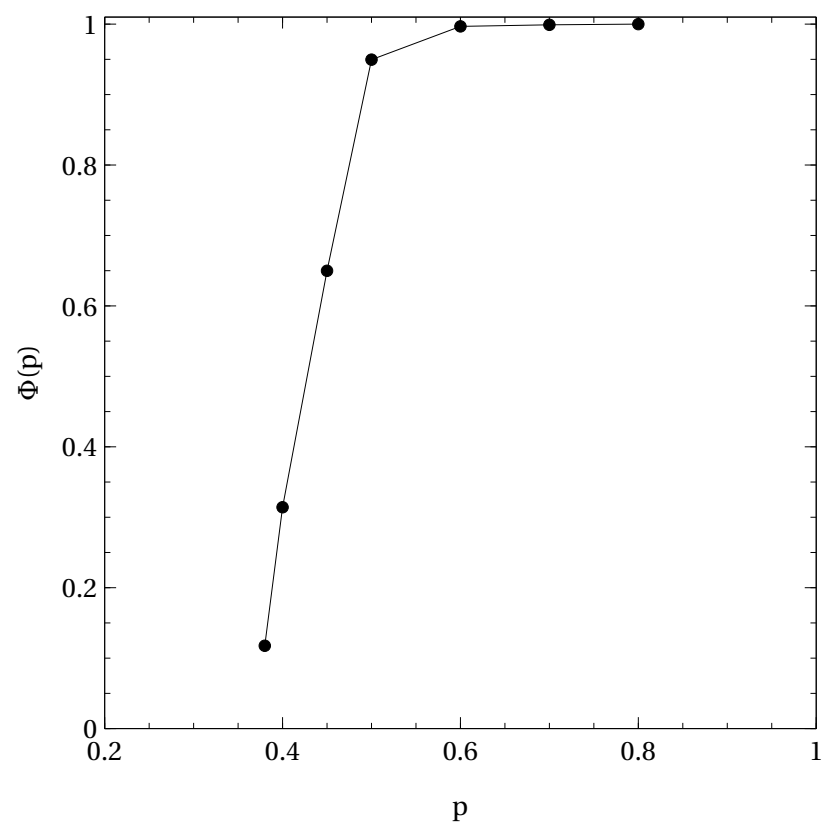

Figure 4: Ratio of delocalized eigenstates w.r.t. all energy eigenstates $\Phi(p)$ as calculated by counting the states between the mobility edges.

Note that this expression is at odds with periodic boundaries, i.e., a (short) transition from one edge of the sample through the the "periodic boundary closure" is, give (10), equivalent to a (long) transition through the whole sample in the opposite direction. Therefore we modify the expression to ensure that only the "shortest" transitions are taken into account. This is achieved by the following definition of the current operator:

$$
\hat{J}=\sum_{i j}^{N} J_{j i} \hat{a}_{j}^{\dagger} \hat{a}_{i}
$$

$J_{j i}=\frac{q}{\hbar} \begin{cases}(j-i) a t_{i j}, & |i-j| a<\frac{L}{2} \\ \operatorname{sign}(j-i)\left([L-(j-i)] a t_{i j}\right) & ,|i-j| a>\frac{L}{2}\end{cases}$

Here $q$ denotes the electric charge per particle, e.g., elementary charge of a single electron. In addition to the current operator we introduce one more quantity, namely the normalized current auto-correlation function $j^{\prime}(t)$, which is better suited for the investigation of finite-size effects and convergence behavior than the actual current auto-correlation. It is given by

$$
j^{\prime}(t)=\frac{\operatorname{Tr}\{\hat{J}(t) \hat{J}(0)\}}{\operatorname{Tr}\left\{\hat{J}^{2}(0)\right\}} .
$$

Numerical results for (12) are displayed in Fig. (5) for various system sizes. (Each curve is the average over 15 runs for different models featuring the same $p$. However 
variations with different individual implementations turn out to be small.) Since the graphs coincide for times where they are significant different from zero for, say, $L \geq 18$, it is justified to assume that at $L=28$ the system is no longer affected by finite-size effects. This conclusion is supported by the observation, that the calculation of (7) reveals a deviation of the results for a system with $L=26$ compared to one with $L=28$ of approximate $0.9 \%$, and continuous decrease of the deviation for larger systems.

At this point a comment on numerical techniques is appropriate. Results up to $L=24$ in this paper are always obtained by numerical matrix diagonalization, whereas all results for sizes above this limitation are calculated by means of a algorithm based on "typicality" that allows for the determination of correlation functions on the basis of propagation of single pure states. In the work at hand the pure state propagation is performed using a standard Runge-Kutta algorithm. For a full account of this typicality technique and its theoretical background, see Refs. $33-35]$. We were able to treat systems up to $L=34(N \approx 39000)$ with this algorithm on standard computing equipment, however as pointed out above, but $L=28$ appears to be sufficient for a reasonable extraction of quantitative results. Nevertheless based on data only from exact diagonalization the whole investigation presented here would have been far less conclusive.

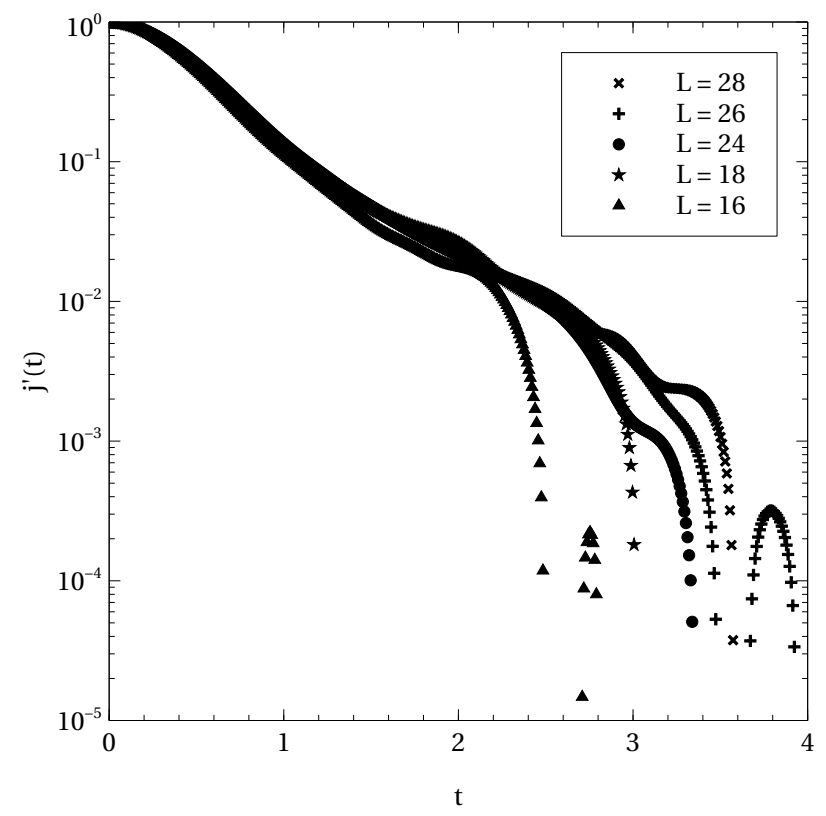

Figure 5: Normalized current auto-correlation functions $j^{\prime}(t)$ for various system sizes $L$. The graphs coincide regardless of size in regions where they are substantially different from zero, for, say $L \geq 18$. Hence data can reliably be expected to contain negligible finite-size effects at $L=28$.

The results on conductivity are shown in Fig. (6)

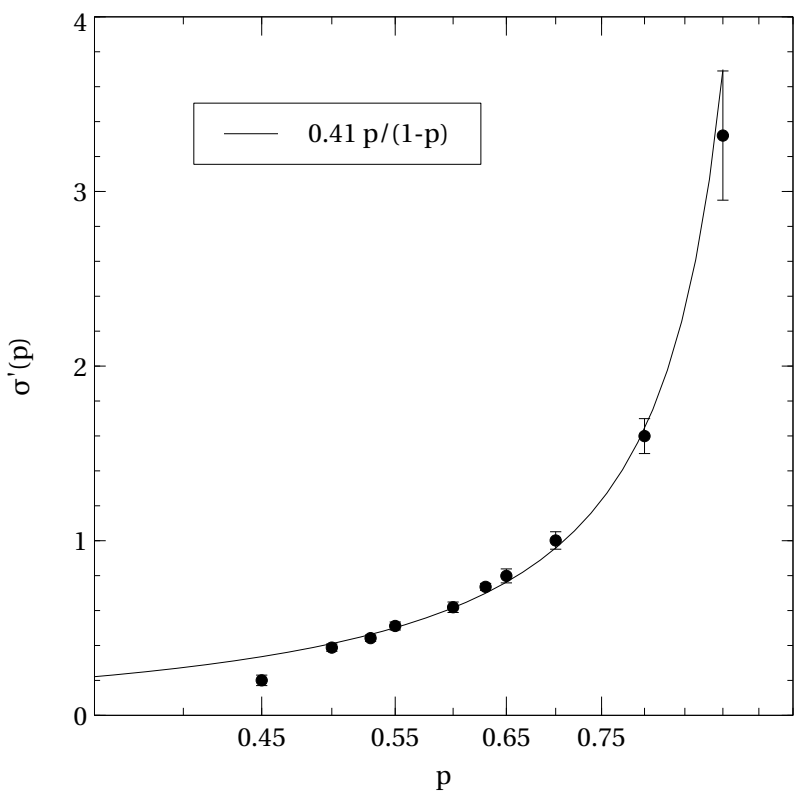

Figure 6: Numerically calculated scaled dc-conductivity $\sigma^{\prime}:=$ $T f^{-1} \sigma_{d c}$ compared to the result of a simple heuristic theory given in the text. The agreement is good, deviations appear at and below $p \approx 0.45$. This is due to non-negligible localization. Note that all data points carry error bars, however for small $p$ they are barely visible.

where $\sigma^{\prime}$ relates to the $\sigma_{d c}$ from (17) as

$$
\sigma_{d c}=\frac{f q^{2} t^{2}}{k_{B} T a \hbar[E]} \sigma^{\prime}
$$

thus $\sigma^{\prime}$ is a dimensionless integrated current autocorrelation function, i.e, $q, \hbar$ are set to unity and $[E]$ is the unity according to which energy is measured. Each conductivity represents the average over 15 different percolation models featuring the same $p$. The error bars indicate the mean square deviation corresponding to the respective 15 conductivities. As expected, the conductivity increases with increasing $p$. A systematic interpretation of this result appears challenging. Nevertheless we want to point out the reasonable agreement of the results displayed in (6) with results from a simple heuristic reasoning. From simple Drude type arguments one expects the conductivity to be proportional to the square of the mean particle velocity $v^{2}$ and the mean collision-free or relaxation time $\tau$, i.e. [36],

$$
\sigma \propto v^{2} \tau
$$

For the square of the mean particle velocity one may simply take $v^{2}:=\operatorname{Tr}\left\{\hat{J}^{2}(0)\right\} / N$. From the current operator as given in (11) it is straightforward to see that his quantity must scale as $p$, i.e., e $v^{2} \propto p$. The relaxation time $\tau$ is (by definition) inversely proportional to a scattering rate $R$. In the percolation model at hand scattering (and thus relaxation of the current) is caused by the "missing 
connections". The number of the latter is proportional to $1-p$, hence one obtains for the rate $R \propto 1 /(1-p)$. Plugging those results into (14) yields

$$
\sigma \propto \frac{p}{1-p}
$$

The solid line in Fig. 6 shows a fit based on (15) yielding $\sigma^{\prime}=0.41 p /(1-p)$. Obviously the agreement is rather good for $p \geq 0.45$. Apparently in this regime the above simple heuristic argument captures the relevant physics, even though below say $p \approx 0.9$ this regime can certainly not be classified as a weak scattering regime. Below $p \approx 0.45$ the fit appears to deviate, however, as may be inferred from Fig. 4, this is the point at which localization massively sets in. We thus conclude that the simple theory given in (15) holds for $p$ down to the quantum percolation threshold. Furthermore it is clearly noticeable that the statistical splay of the results increases with increasing $p$. This however may be readily interpreted as a consequence of the law of large numbers: the fewer scattering centers there are the larger is the statistical variation of all quantities that depend on scattering.

Next we consider the specific kind of decay of the current auto-correlation function. As shown in Fig. (17) a transition of transport types appears to occur between $p=0.9$ and $p=0.6$. Decay at $p=0.9$ is compared to a mono-exponential decay, as to be expected from a simple Drude model or a linear Boltzmann equation in relaxation time approximation [25, 36, 37], the agreement is reasonable. At $p=0.65$, however, the decay behavior is much closer to a Gaussian as illustrated in Fig. (7). This transition from exponential to Gaussian decay behavior on the way from weak to strong scattering has been observed in various other, similar models before. There it has been explained within the framework of a timeconvolutionless projection operator investigation [15, 38]. If one projects onto the current and performs a perturbative, leading order treatment, then exponential decay of the current auto-correlation function results at weak, and Gaussian decay at intermediate strength perturbations. From the results displayed in Fig. (7) it appears evident that the same applies to the model at hand as well.

\section{EINSTEIN RELATION AND MEAN FREE PATH}

The diffusion constant of a system is certainly interesting in its own right. However the main purpose of this section is to establish the validity of an Einstein relation in oder to arrive at a reasonable definition of a mean free path. The validity of the Einstein relation in quantum systems is frequently discussed [39, 40]. Here we examine it in its most elementary form, namely as the claim of a proportional relation between conductivity and diffusion

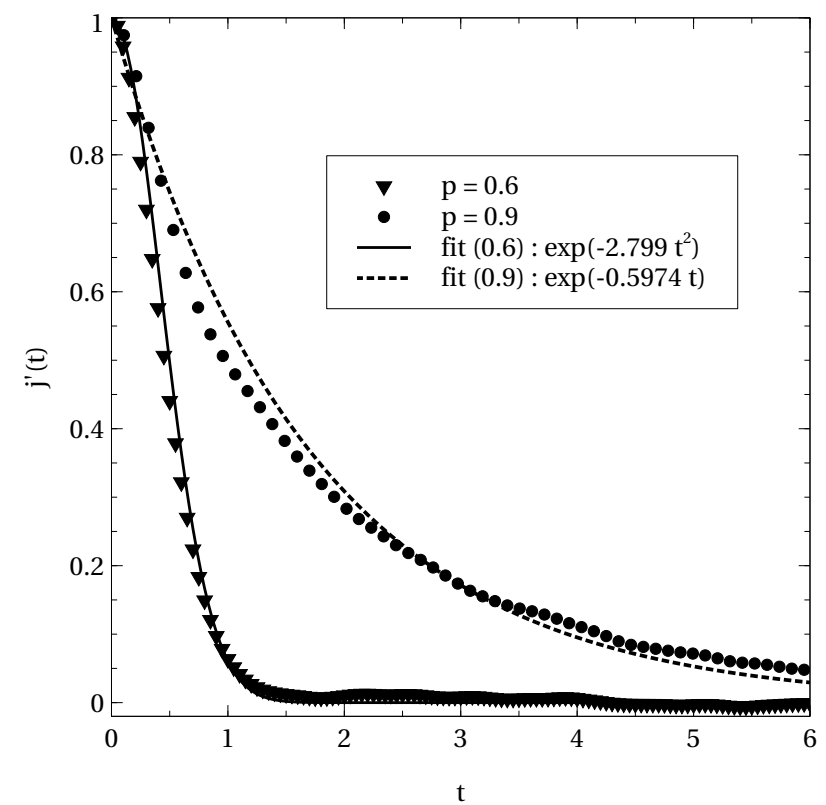

Figure 7: Normalized current auto-correlation functions $j^{\prime}(t)$ at $L=28$ for $p=0.9$ (few defects) and $p=0.6$ (medium defects). At $p=0.6$ the decay appears to be Gaussian, whereas at $p=0.9$ one finds rough agreement with an exponential decay. The latter hints in the direction of Drude-type transport.

constant

$$
D(t)=\frac{T}{\epsilon^{2}} \sigma(t)
$$

where $D(t)$ denotes the (time dependent) diffusion constant, $\epsilon^{2}$ denotes the uncertainty (variance) of the transported quantity per site at equilibrium. Since the uncertainty for the dc-current equals at low densities the filling factor $f$, cf. [12], we find from linear response theory (7)

$$
D_{K}(t)=\int_{0}^{t} \frac{1}{N} \operatorname{Tr}\left\{\hat{J}\left(t^{\prime}\right) \hat{J}(0)\right\} d t^{\prime} \quad .
$$

$D_{\mathrm{K}}(t)$ is to be compared to a direct computation of the diffusion constant in order to check (16). If a diffusion equation holds, the derivation w.r.t. time of the spatial variance of the diffusing quantity equals twice the diffusion constant [1].

To directly observe this spatial variance we define an initial density operator

$\hat{\rho}(0)=\frac{1}{Z} \exp \left(-\frac{\left(\hat{x}-\frac{L}{2}\right)^{2}}{2 d}\right), \quad Z=\operatorname{Tr}\left\{\exp \left(-\frac{\left(\hat{x}-\frac{L}{2}\right)^{2}}{2 d}\right)\right\}$,

where $d$ denotes an initial variance, which is here chosen as $d=0.95$.

This implies that the initial site occupation probability is concentrated in a thin slab of a thickness on the order of one perpendicular to the $\mathrm{x}$-axis.

Based on this $\hat{\rho}(0)$ we calculate the time-depended vari- 
ance and take the derivative w.r.t. time to obtain a diffusion constant; here named $D_{\mathrm{D}}(t)$.

$$
D_{D}(t)=\frac{1}{2} \frac{d}{d t} \operatorname{Tr}\left\{\hat{x}^{2}(t) \rho(0)\right\}
$$

Note that, since the mean particle position does not drift $\frac{d}{d t} \operatorname{Tr}\{\hat{x}(t) \rho(0)\}$ remains without influence.

If the Einstein relation holds, $D_{\mathrm{K}}(t)$ and $D_{\mathrm{D}}(t)$ should coincide. Fig. (8) shows the comparison of both diffusion constants and reveals that the Einstein relation is apparently fulfilled. Moreover it is obvious that the calculation of the diffusion constant in the sense of (19) is strongly influenced by finite-size effects.

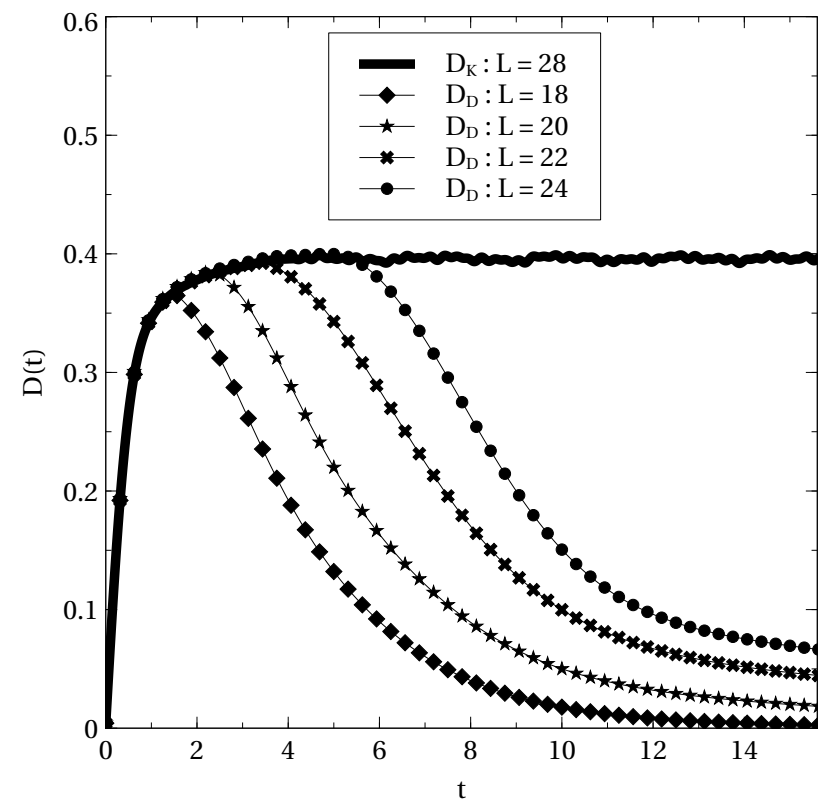

Figure 8: Comparison of time-dependent diffusion constants either calculated by (17) or (19) at $p=0.5$. The calculation according to (19) obviously suffers from strong finite-size effects, however, agreement in the limit of large sizes is evident. This indicates the validity of the Einstein relation.

However, the validity of the Einstein relation allows for a reasonable definition of a mean free path $\lambda$ which may be calculated based on (17).

Ballistic transport behavior, as exhibited by initially concentrated, free, non-scattering particles is characterized by a quadratic increase of the spatial variance w.r.t. time, i.e., $\left\langle\hat{x}^{2}\right\rangle \propto t^{2}$ or $D \propto t$, Since the increase of the diffusion constant is linear in the beginning, cf. Fig. (8), we define the increase of standard deviation $\sqrt{\left\langle\hat{x}^{2}\right\rangle}$ during this ballistic initial period as the mean free path $\lambda$. We define the ballistic initial period as the period before $D(t)$ has reached $90 \%$ of its final value. Of course this choice is not imperative, however from looking at Fig. (8) it appears reasonable. Fig. (9) shows the results for the mean free path $\lambda$

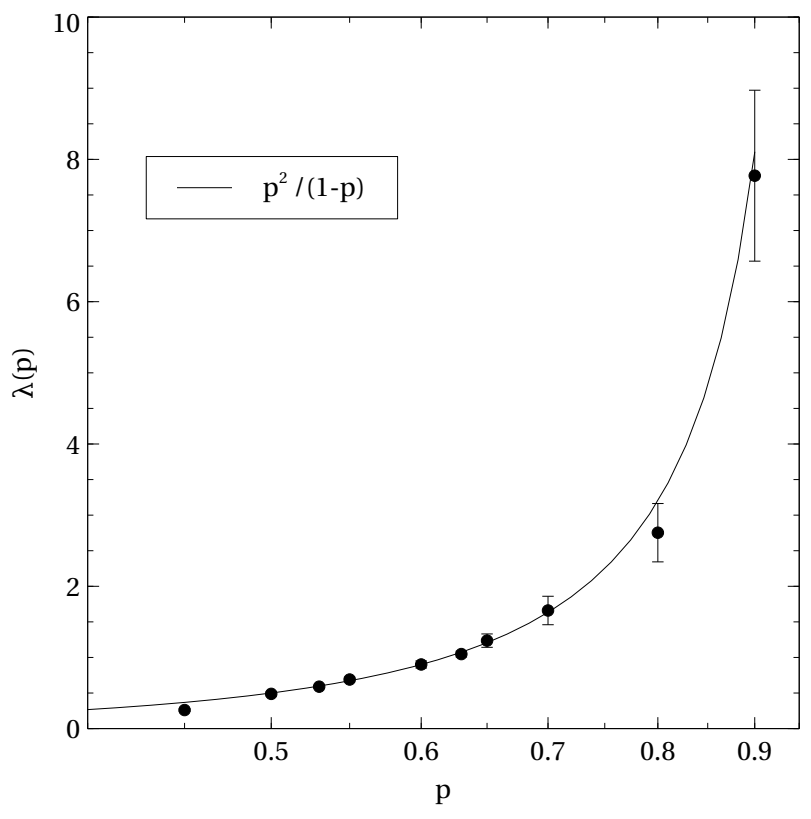

Figure 9: Numerically calculated mean free path $\lambda$ compared to the result of a simple heuristic theory given in the text. The agreement is good, deviations appear below $p \approx 0.5$. This is due to non-negligible localization. Note that all data points carry error bars, however for small $p$ they are barely visible.

Much like the consideration on conductivity in Sec. IV] we discuss the agreement of a simple, heuristically derived form of $\lambda$ with the computed data in Fig. (9) in the following. Consider some "chain" of either connections or voids along some crystal-axis. Assume, for simplicity that this chain was ordered (which it is in fact not). Assume furthermore that a longer sequence of connections alternates with just one void. Call the length of the sequence of connections $l$. Then the total ratio $p$ of connections per total number of sites is $p=l / l+1$. Or the length of the uninterrupted sequence of connections depends on the connection probability as:

$$
l(p)=\frac{p}{1-p}
$$

We may associate $l$ with a free path. I order to find the mean free path we multiply $l$ by $p$ since this is the probability (relative frequency) of a "site" to sit on a sequence of connections. We thus get:

$$
\lambda(p)=\frac{p^{2}}{1-p}
$$

This expression for the mean free path is represented in Fig. (9) by the solid line. Given the simplicity of the argument the agreement with the computed data is good. Of course such an expression can only be expected to yield reasonable results down to $p=0.5$. However from Fig. (8) we now that below that localization effects set in anyway. Thus for the fully delocalized regime (21) appears to capture the relevant physics. 


\section{SEMI-QUANTITATIVE COMPARISON OF THE RESULTS TO MEASURED CONDUCTION DATA ON BINARY ALLOYS}

As already pointed out in the Introduction the primary intentions of the paper at hand are of principal nature. However a short comment on the relation of the results to conductivities of binary alloys should be in order. The electronic system of a binary alloy in a mixed crystal phase may be viewed as an implementation of a percolation model. If the valency of the solute element is very different from that of the host metal, the on-site potentials at the solute sites may be so low (high) that, as a rough approximation, the solute sites may be regarded as being "frozen out", i.e., not contributing to the conduction process. Such a picture suggests a site percolation rather than a bond percolation model, but since bond and site percolation are expected to behave more or less comparably we simply ignore this difference in this consideration. The conductivity of weakly or non-interacting fermions at rather low temperatures $\left(k_{B} T\right.$ small compared to the bandwidth) is roughly given by

$$
\sigma_{f} \approx \frac{n\left(E_{f}\right)}{N} \int_{0}^{t} \frac{1}{\operatorname{Tr}\left\{\hat{P}_{f}\right\}} \operatorname{Tr}\left\{\hat{J}\left(t^{\prime}\right) \hat{J}(0) \hat{P}_{f}\right\} d t^{\prime}
$$

25] where $n\left(E_{f}\right)$ is the density of states at the Fermi energy, $N$ is the total number of states in the conduction band of the one-particle model and $\hat{P}_{f}$ is a projector which projects onto an energy shell (Hilbert space spanned by energy eigenstates) of width $k_{B} T$ around the Fermi energy. Separating dimensionless quantities from quantities carrying dimensions yields

$$
\sigma_{f} \approx \frac{n\left(E_{f}\right) q^{2} t^{2}}{N a \hbar[E]} \sigma_{f}^{\prime}
$$

where $\sigma_{f}^{\prime}$ is the corresponding dimensionless current auto-correlation function, just like $\sigma^{\prime}$ in (7). Since we are only doing an estimate we replace $\sigma_{f}^{\prime}$ by $\sigma^{\prime}$ as given in Fig. [6] i.e., $\sigma_{f}^{\prime} \approx 0.41 p / 1-p$. If the percentage of solute atoms $c[\%]:=100(1-p)$ is low we may approximate $\sigma_{f}^{\prime} \approx 41 / c$. We intend to compare our results to recently measured data on magnesium alloys, specifically a magnesium-zirconium alloy [18]. In order to do so we use the following values in (23): The bandwidth of metallic magnesium is ca. $14 \mathrm{eV}$ [2], our simple cubic basis model yields a bandwidth of ca. $14 \mathrm{eV}$ if the hopping terms are chosen as $t=1.2 \mathrm{eV}$. (Obviously we use $\mathrm{eV}$ as an energy unit). According to [42] we furthermore set the relative density of states to $n\left(E_{f}\right) / N \approx 0.16 / e V$. Since our model does not account for any lattice distortions, the lattice constant is set to $a=3 A$ which is about the mean lattice constant of metallic magnesium. And, naturally, the transported charge per particle is the electron charge, i.e., $q=e$. Plugging in all these numbers and calculating the specific electrical resistivity $\rho=1 / \sigma_{f}$ rather than the conductivity itself, we get

$$
\rho \approx c \cdot 1.3 \cdot 10^{-7}(\Omega m)
$$

Of course this cannot be taken as an absolute result since even pure magnesium $(c=0)$ has a non-zero resistivity due to phonons, impurities, etc. But if one, as suggested by Matthiessen's rule, regards (24) as an expression for the increase of the resistivity due to the gradual addition of a a solute, (24) may be compared to experimental data. Pan et al. report in Ref. [18] for a magnesium-zirconium alloy a value of

$$
\rho_{\text {measured }}=c \cdot 9.311 \cdot 10^{-8}(\Omega m)
$$

The atomic volume difference between magnesium and zirconium is rather low, such that few lattice distortions can be expected. Furthermore the valency of zirconium $(+4)$ is rather high. However, note that our model has simple cubic rather than hexagonal symmetry, we consider bond rather than site percolation, the concept of zirconium sites being frozen out is surely not completely correct, we neglect lattice distortions entirely, etc. Regarding all these limitations the agreement of (24) with (25) within about $30 \%$ appears reasonable.

\section{SUMMARY AND CONCLUSION}

We investigated a simple quantum bond percolation model on the basis of an one-particle, tight-binding Hamiltonian. We focus on investigation of transport properties in the fully delocalized regime, i.e., a regime in which only a negligible fraction of all energy eigenstates is localized. This turns out to be the case at bond probabilities of $p \geq 0.5$. The conductivity in this regime has been calculated using linear reponse theory (Kubo-formula) and a numerical algorithm based on quantum typicality for the evaluation of the current autocorrelation function. As expected the conductivity increases rapidly with increasing $p$ and is found to be in accord with the result of a simple heuristic reasoning involving mean collision free times and mean particle velocities. The latter may be defined even though at $p \geq 0.5$ no true dispersion relations exist. Furthermore a gradual transition from a current decay that is not in accord with a Drude model to a current decay that is, is observed between $p \approx 0.6$ and $p \approx 0.9$. The proportionality of the conductivity and the diffusion constant, i.e., the Einstein relation is analyzed numerically and found to hold. This finding allows for a definition of a mean free path. Numerical calculations of this mean free path coincide well with results from yet another heuristic consideration based on counting the mean length of uninterrupted sequences of connections in the lattice. Thus, to conclude, in the regime above $p=0.5$, although being fully quantum and strongly disordered, the dynamics of the model appear to be remarkably well described by by purely probabilistic, classical reasoning. Furthermore the result based on the percolation model are in reasonable agreement with measured data on binary magnesium alloys. 
[1] B. Shapiro, Percolation Structures and Processes, vol. 5 (Annals of the Israel Physics Society, 1983).

[2] S. Kirkpatrick, Rev. Mod. Phys. 45, 574-588 (1973).

[3] D. H. Lee, Phys. Rev. B 50, 10788 (1994).

[4] A. Sanpera, A. Kantian, L. Sanchez-Palencia, J. Zakrzewski, and M. Lewenstein, Phys. Rev. Lett. 93, 040401 (2004).

[5] R. Yu, T. Roscilde, and S. Haas, Phys. Rev. Lett. 94, 197204 (2005).

[6] A. Kaneko and T. Ohtsuki, Journal of the Physical Society of Japan 68, 1488 (1999).

[7] P. W. Anderson, Phys. Rev. 109, 1492-1505 (1957).

[8] C. M. Soukoulis, Q. Li, and G. S. Grest, Phys. Rev. B 45, 7724 (1992).

[9] G. Schubert, A. Weisse, and H. Fehske, Phys. Rev. B 71, 045126 (2005).

[10] C. D. Lorenz and R. M. Ziff, Phys. Rev. E 57, 230 (1998).

[11] R. Berkovits and Y. Avishai, Phys. Rev. B 53, 16125 (1996).

[12] N. Hashitsume, M. Toda, R. Kubo, and N. Saito, Statistical physics II: nonequilibrium statistical mechanics (Springer, 1992).

[13] P. Gaspard, Phys. Rev. E 53, 4379 (1996).

[14] R. Steinigeweg, J. Gemmer, H.-P. Breuer, and H.-J. Schmidt, Eur. Phys. J. B 69, 275 (2009).

[15] R. Steinigeweg, H. Niemeyer, and J. Gemmer, New J. Phys 12, 113001 (2010).

[16] U. Schneider and L. Hackmüller, Nature Physics 8, 213 (2012).

[17] L. Hackmüller and U. Schneider, Science 327, 1621 (2010).

[18] H. Pan and F. Pan, Journal of material science 49, 3107 (2014).

[19] C. M. Soukoulis and G. S. Grest, Phys. Rev. B 44, 4685 (1991).

[20] E. Abrahams, 50 Years of Anderson Localization (World Scientific Publishing Company, 2010).

[21] D. J. Priour, Phys. Rev. B 85, 014209 (2012).

[22] I. Mertig, E. Mrosan, and P. Ziesche, Multiple Scattering Theory of Point Defects in Metals: Electronic Properties
(Teubner-Texte zur Physik, 1987), 11th ed.

[23] J. Brndiar and P. Markos, Phys. Rev. B 74, 153103 (2006).

[24] L. Ujfalusi and I. Varga, Quantum percolation transition in 3d (2014), URL http://arxiv.org/pdf/1405.1985.

[25] J. Jaeckle, Einführung in die Transportheorie (Vieweg, 1978).

[26] R. Kubo, J. Phys. Soc. Jpn. 12, 570 (1957).

[27] R. Zwanzig, Annual Review of Physical Chemistry 19, 67 (1965).

[28] R. Kubo, Rep. Prog. Phys. 29, 255 (1966).

[29] F. Heidrich-Meisner, A. Honecker, D. C. Cabra, and W. Brenig, Phys. Rev. B 68, 134436 (2003).

[30] J. Gemmer, R. Steinigeweg, and M. Michel, Phys. Rev. B 73, 104302 (2006).

[31] J. Benz, T. Fukui, A. Klümper, and C. Scheeren, J. Phys. Soc. Jpn. 74, 181 (2005).

[32] A. Khodja, H. Niemeyer, and J. Gemmer, Phys. Rev. E 87, 052133 (2013).

[33] T. A. Elsayed and B. V. Fine, Phys. Rev. Lett. 110, 070404 (2013).

[34] R. Steinigeweg, A. Khodja, H. Niemeyer, C. Gogolin, and J. Gemmer, Phys. Rev. Lett. 112, 130403 (2014).

[35] R. Steinigeweg, J. Gemmer, and W. Brenig, Phys. Rev. Lett. 112, 120601 (2014).

[36] N. W. Ashcroft and N. D. Mermin, Solid State Physics (Saunders College Publishing, New York, 1976).

[37] G. D. Mahan, Many-Particle Physics (Springer, 2000), 3rd ed.

[38] R. Steinigeweg and R. Schnalle, Phys. Rev. E 82, 040103 (2010).

[39] R. Steinigeweg, H. Wichterich, and J. Gemmer, Europhys. Lett. 88, 10004 (2009).

[40] Y. Roichman and N. Tessler, Appl. Phys. Lett. 80, 1948 (2002).

[41] A. Einstein, Annalen d. Physik p. 549 (1905).

[42] S. Chatterjee and P. Sinha, J. Phys. F: Met. Phys. 5, 2089 (1975). 